\title{
The solar system between order and chaos
}

\author{
Stefano Marmi
}

Published online: 28 May 2013

(C) Centro P.RI.ST.EM, Università Commerciale Luigi Bocconi 2013

\begin{abstract}
Poincaré's fertile error reintroduced the oldest open question in dynamical systems: the problem of stability of orbits in the n-body problem. After Poincare the problem was studied by Birkhoff, Kolmogorov, Arnold and Moser. Laskar, using the secular system introduced by Lagrange, showed that the orbits of the planets in a realistic model of the solar system are chaotic with a Lyapounov exponent of $1 /(5 \mathrm{Myrs})$.
\end{abstract}

Keywords Henri Poincaré - Three-body problem . Celestial mechanics - Chaos theory - Dynamical systems . Planetary orbits

Henri Poincaré's fertile error marked the demise of the dream that had guided mathematical research for more than two centuries: gone was the possibility of exactly describing and predicting the dynamics of any deterministic system, whatever its initial state might be. The three-body problem was not integrable [1], that is, its solution could not be traced back to an analytical-algebraic procedure of 'quadrature'. The procedure classically used to solve simpler differential equations was supposed to have made it possible, not only in principle but also computationally, to predict with a predefined degree of precision the future position of planets.

The problem of the stability of planetary orbits in the solar system, believed to have been solved for some time,

S. Marmi ( $\square)$

Scuola Normale Superiore di Pisa, Piazza dei Cavalieri,

7, 56126 Pisa, Italy

e-mail: stefano.marmi@sns.it

S. Marmi

CNRS UMI 3483 - Laboratorio Fibonacci,

Piazza dei Cavalieri, 7, 56126 Pisa, Italy presented itself again in its entirety. Successive to the work of Poincaré, George David Birkhoff showed that the instability of planetary motion was indeed possible, and that the structure of the orbits in the phase space of the $n$ body problem was formidably complex.

An important step forward was taken in 1954 by Andrei Nikolaevich Kolmogorov, with the statement of the theorem of persistence of quasiperiodic orbits in quasi-integrable Hamiltonian systems. Later proved by Vladimir Arnold and Jürgen Moser, it became widely known as the KAM theorem. Kolmogorov's result was effectively a vindication of the paradigm of the stability and predictability of orbits in the problems of celestial mechanics: in fact, the quasiperiodic motions are indistinguishable from periodic motions if the precision of the observations is fixed and finite. However, in contrast to a genuine periodic motion, in the case of quasiperiodic motion the observed period is increased limitlessly to improve precision. As opposed to genuinely instable and chaotic behaviours predicted in the work of Poincaré and Birkhoff, quasiperiodic orbits do not manifest any sensitive dependence on initial data and the construction of reliable ephemerides is presumed to be possible, at least in some cases.

Kolmogorov, who was very familiar with Poincaré's work as well as that of Birkhoff, suggested that the typical structure of orbits in the phase space of a Hamiltonian system would indicate the co-existence of an instable behaviour for a topologically generic set of initial conditions, and a stable and quasiperiodic behaviour for a probabilistically generic set of initial conditions.

The instability of a topologically generic set of initial conditions implies that, even if the motion of a planet or an asteroid is regular, an arbitrarily small perturbation of its position or velocity is sufficient to send it into an orbit that is irregular and chaotic ('Arnold diffusion'). The process 
with which this type of diffusion can manifest itself is however rather slow (Nekhoroshev's theorem) and this set of results, although of a theoretical nature and not directly applicable to realistic models of the solar system, strengthened the conviction that the time scale necessary for a chaotic behaviour to manifest itself in the dynamics of the solar system had to be very long in comparison to its age.

The numerical simulations of the dynamics of the solar system ran up against the necessity of integrating the Newton equations for the $n$-body problem for billions of years with a very brief step of temporal integration, of a few hours at most. In fact, the fastest planet, Mercury, carries out its orbital motion in $<3$ months, and using longer steps of integration would lead to errors in the numerical schemes capable of invalidating the conclusions.

In 1990 Jacques Laskar got around the problem by substituting the so-called secular system introduced by Lagrange in place of the system of Newton equations. The main advantage of this procedure consists in the elimination of the fast angular variables (which correspond to unperturbed motion of the planets along Keplerian ellipses). This makes it necessary to renounce the calculation of the ephemerides, limiting the study to the variables that describe the dynamics of the slow deformation of the orbits of the planets in time as a result of reciprocal perturbations.

In reality, the variable used by Laskar were introduced by Poincaré: the unperturbed motion of the planets corresponds simply to a point of equilibrium for the dynamics of the secular system. Since in these variables the Keplerian solutions correspond to the absence of motion, it is possible to use a step of integration much longer than that necessary for the direct numerical simulation of the system: 500 years instead of a few hours.

This made it possible for the first time to numerically integrate a realistic model of the solar system on a temporal scale of the order of $10^{10}$ years. The results were surprising [2]: the orbits of the planets of the internal solar system (Mercury, Venus, Earth and Mars) are chaotic with a temporal scale for instability, measured in Lyapounov time, on the order of five million years. A practical consequence of such a low value (on a scale of astronomical time) of the
Lyapounov time is the impossibility of calculating a planetary ephemeris for the internal planets: in some 100 million years an error of $15 \mathrm{~m}$ in the initial position could grow into an error on the scale of 150 million kilometres, that is, equal to the actual distance of the Earth to the Sun. The situation is quite different in the case of the external solar system: the orbits of the larger planets (Jupiter, Saturn, Uranus and Neptune) appear to be described well by quasiperiodic motions. Intense research work to prove this result rigorously is ongoing in Italy, with mathematical physicists Antonio Giorgilli and Ugo Locatelli taking an active part.

Translated from the Italian by Kim Williams

\section{References}

1. Chenciner, A.: Poincare and the Three-Body Problem. Séminaire Poincaré (Bourbaphy). XVI, 45-133 (2012). Available at http://www.bourbaphy.fr/novembre2012.html. Accessed 01 April 2013

2. Marmi, S.: Chaotic behaviour in the solar system (following J. Laskar). Astérisque 266, 113-136 (2000)

\section{Author Biography}

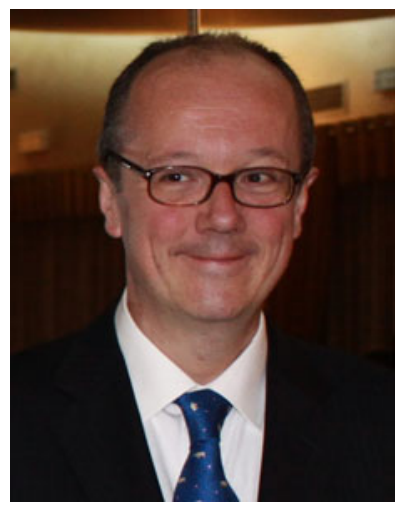

Stefano Marmi is Professor Ordinario of Dynamical Systems at the Scuola Normale Superiore di Pisa. Before this, he was a Ricercatore at the University of Florence, and then Professore Associato at the University of Udine. He studied physics at the University of Bologna, and received his Ph.D. in theoretical physics there in 1990. He was awarded the ISAAC prize in 1999. He has spent long periods of research at the departments of mathematics of the Freie Universität in Berlin and the Université de Paris-Sud (Orsay). He is the author of Analytical Mechanics, Oxford University Press (with A. Fasano, Bollati Boringhieri, Torino, 2nd ed., 2002). He has published numerous articles in peer-reviewed journals. 